Original Article

\title{
PREPARATION AND CHARACTERIZATION OF ORAL NANOSUSPENSION LOADED WITH CURCUMIN
}

\author{
SNEHA DEKATE 1 , SRINIVAS BHAIRY2 ${ }^{2}$, RAJASHREE HIRLEKAR ${ }^{* 3}$ \\ 1,2,3Department of Pharmaceutics, Vivekanand Education Society's College of Pharmacy, Hashu Advani Memorial Complex, Behind \\ Collector Colony, Chembur (E), Mumbai 400074. Maharashtra, India \\ Email: rajashree.hirlekar@ves.ac.in
}

Received: 15 Aug 2017 Revised and Accepted: 08 May 2018

\begin{abstract}
Objective: The principle objective of the present research work was to improve the bioavailability of curcumin (CUR) by decreasing its particle size. Nanosuspension (NS) of CUR was prepared using poloxamer-188 (P188) as a surfactant. The prepared NSs were characterized for particle size, polydispersity index (PDI), zeta potential, drug loading, saturation solubility, and drug release kinetic studies.

Methods: Components required for NS preparation, such as solvent, anti-solvent and surfactant were screened. Precipitation high-speed homogenization (HSH) method was used for the preparation of NS using selected components. Evaluation of NS for particle size, PDI, drug loading, saturation solubility and in vitro drug release was done. Pharmacokinetic studies of the NS in sprague dawley (SD) rats were performed.

Results: The particle size, PDI and zeta potential of the optimized formulation was $596.5 \pm 5 \mathrm{~nm}, 0.233 \pm 0.010$ and $-23 \pm 2 \mathrm{mV}$ respectively. The pH of all the formulations was in the range of 5-6 which is acceptable when related to drug stability. The optimized formulation showed an increase in saturation solubility in water and phosphate buffer $\mathrm{pH} 6.8$ when compared to plain CUR suspension (S). Results of pharmacokinetic studies indicated that Cmax and $\mathrm{AUC}_{0-6}$ were increased 8 and 10 times respectively from plain CUR S to CUR NS.
\end{abstract}

Conclusion: CUR NS was prepared using P188 as the stabilizer. Amongst various stabilizers screened P188 rendered a stable NS with the particle size in nano range. Pharmacokinetic studies revealed the better performance of CUR NS as compared to plain CUR S.

Keywords: Curcumin, High-speed homogenization, Probe sonicator, Pharmacokinetic studies

(C) 2018 The Authors. Published by Innovare Academic Sciences Pvt Ltd. This is an open access article under the CC BY license (http://creativecommons.org/licenses/by/4.0/) DOI: http://dx.doi.org/10.22159/ijpps.2018v10i6.22027

\section{INTRODUCTION}

Cancer is one of the most devastating diseases that involve various cellular and genetic alterations [1], a leading cause of mortality. It strikes more than one-third of the world's population and it is the cause of more than $20 \%$ of all deaths. Among the causes for cancer are tobacco, viral infection, chemicals, radiation, environmental factors and dietary factors. Surgery, chemotherapy and radiotherapy are the main conventional cancer treatment often supplemented by other complementary and alternative therapies [2].

Currently, there are a limited number of formulation approaches available for compounds that are poorly soluble in water. The most direct approach for enhancing compounds solubility is to generate a salt. If, however, the compound is non-ionizable, solubility concerns are generally addressed by micronization and/or the development of oil-based solutions in gelatin capsules, i.e. soft-gel technology. In addition, co-solvents, surfactants or complexing agents such as cyclodextrins $[3,4]$ have been employed. Reasonable success has also been met in formulating water-insoluble drugs using emulsion, microemulsion and solid dispersion technology. Although some of these approaches have been successfully utilized, especially for highly potent compounds with low dose requirements, there is a growing need for more effective and versatile ways to handle formulation issues associated with poorly-water-soluble molecules.

Conventional anticancer drugs suffer because of their low aqueous solubility, poor physical stability, reduced absorption, rapid metabolism and instability under highly acidic conditions. To combat these constraints intense research is focused on various sources to develop novel anti-cancer drugs. Plants have proved valuable natural anti-cancer therapy source. Increasing number of cancer patients currently use complementary and alternative medicines in conjunction with conventional chemotherapeutic treatments. The efficacy of many herbal drugs is often limited by their unspecific site of action. Novel drug delivery systems (NDDS) are currently under development so as to lessen drug degradation, reduce dosage and its toxicity, increase solubility and stability. NDDS are advantageous in delivering the herbal drug at a predetermined rate and exhibit site-specific action [1].

NSs are one of the approaches to increase the dissolution rate of practically insoluble drug substances. NSs can be prepared by two methods namely, "bottom-up technology" and "top-down technology" and stabilized by a suitable stabilizer. Bottom-up technology, a method to form nanoparticles utilizes techniques such as precipitation, microemulsion, and melt emulsification [5].

Top-down technology involves the disintegration of larger particles into nanoparticles, examples of which are high-pressure homogenization and milling methods [6]. NS enhance solubility of drugs that are poorly water-soluble and poorly lipid soluble by increase in surface area following particle size reduction, increase in saturation solubility, increase in the time available for dissolution because of inherent adherence characteristics of nanoparticles to the gastrointestinal wall (high specific surface area being indicative of a high interactive potential with biological surfaces) [7].

A new drug substance fails the formulation development process due to very low solubility in water and bioavailability issues. CUR, a hydrophobic polyphenol compound derived from the rhizome of the herb Curcuma longa (Family: Zingiberaceae) is a promising anticancer agent that possesses the ability to inhibit the growth of wide range of human tumors. Various approaches have been tried to improve its solubility, low plasma and tissue level, rapid metabolism and degradation. CUR is a bis- $\alpha, \beta$ unsaturated $\beta$-diketone, (commonly called diferuloylmethane), which exhibits keto-enol tautomerism having a predominant keto form in acidic and neutral solutions and stable enol form in alkaline medium. Commercial CUR contains approximately $77 \%$ diferuloylmethane, $17 \%$ demethoxy-curcumin, and $6 \%$ bisdemethoxycurcumin. CUR undergoes extensive reduction, most likely through alcohol dehydrogenase, followed by conjugation. It 
has been shown to have anticancer activity, and has a potential for interfering with tumor initiation, tumor promotion, angiogenesis and metastasis but shows low plasma and tissue levels due to poor absorption, rapid metabolism, and rapid systemic elimination [8]. Thus bioavailability of the drug can be improved using nanoparticulate drug delivery system such as NS.

\section{MATERIALS AND METHODS}

\section{Materials}

CUR was obtained as gift sample from VAV Life Sciences, India. Stabilizers sample was obtained from BASF, Mumbai. All other chemicals were of analytical grade and HPLC grade as per their use in the research work.

\section{Methods}

\section{Preparation of nanosuspension}

Various components associated with NS formulation were selected after initial screening. These were a suitable solvent phase for drug, stabilizers and its concentration and homogenization speed. CUR NS was prepared by the precipitation-high speed homogenization (HSH Ultraturrax T-25 IKA) method (fig. 1) [6, 7]. Briefly, a solution of the drug in the solvent was slowly introduced into antisolvent phase containing a surfactant, at 1:15 solvent: antisolvent ratio at homogenization speed of $10,000 \mathrm{rpm}$ for $10 \mathrm{~min}$. Then, the mixture was magnetically stirred for $4-5 \mathrm{~h}$ to remove the organic solvent. After the complete evaporation of the solvent, the samples were immediately transferred to a vial and subjected to probe sonication (Oscar ultrasonic Co. Ltd., India) at $90 \mathrm{~W}$ for $10 \mathrm{~min}$. During the process, the temperature was controlled using a water bath.

\section{Screening of organic solvents}

Various water-miscible organic solvents like ethanol, ethyl acetate and acetone were screened to select a suitable solvent for the drug. Excess CUR was added into an organic solvent to form the saturated solution. Solvent dissolving maximum CUR was selected as the solvent phase for further studies.
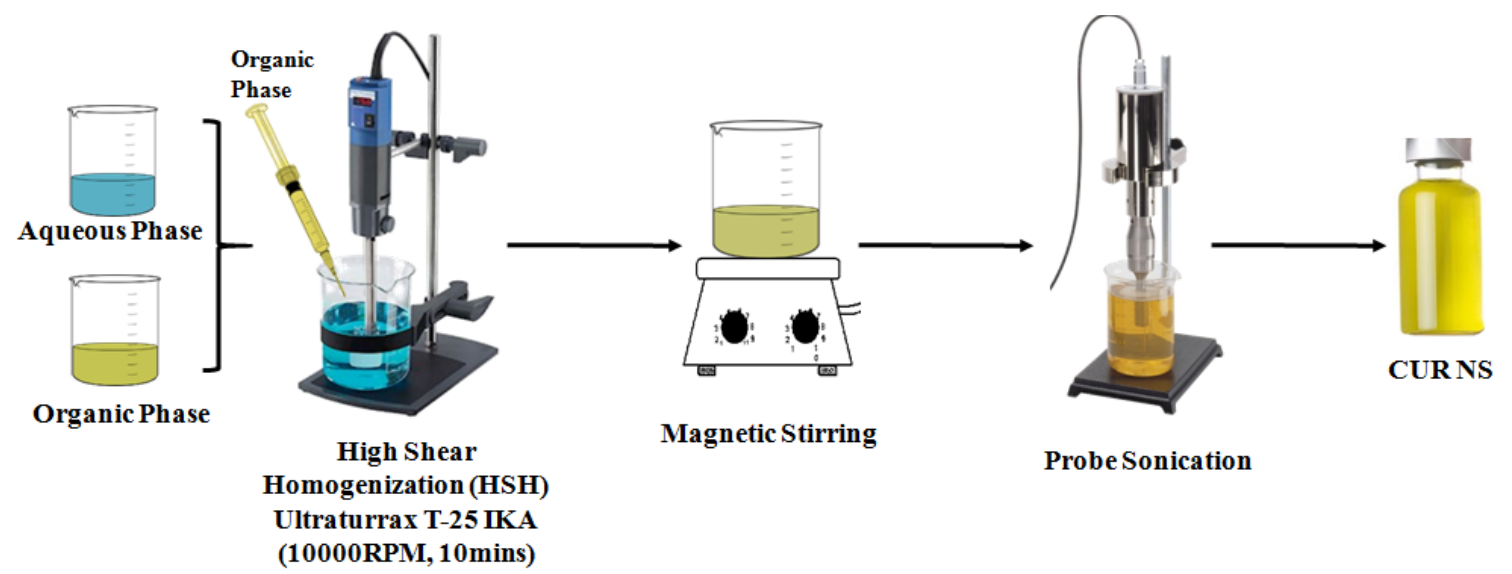

Magnetic Stirring

Probe Sonication

Ultraturrax T-25 IKA

Fig. 1: Schematic representation of the preparation of CUR NS

\section{Screening of stabilizers}

Various surfactants like sodium lauryl sulphate (SLS), tween 80 , d- $\alpha$ tocopherol polyethene glycol 1000 succinate (TPGS 1000) and P188 were screened as stabilizers. These surfactants were dissolved in the organic phase (solvent phase) or distilled water (antisolvent phase) considering their solubility. A suitable quantity of CUR was dissolved in organic solution. The organic solution was slowly injected into antisolvent phase with help of syringe and bath sonicated up to 30 min for precipitation of drug to take place. The organic solvent was evaporated using magnetic stirrer. Particle size was measured. Among various surfactants screened the one conferring maximum stability to the NS was selected. The same procedure was followed, varying concentration of stabilizers to arrive at its appropriate concentration.

\section{Optimization studies}

After initial screening, further optimization was done by varying formulation and process parameters as independent variables. The formulation parameters varied were surfactant concentration and solvent: anti-solvent ratio and the process parameter varied was homogenization speed. Dependent variables measured were particle size and PDI.

Optimization was done using factorial design, in which concentration of surfactant $0.5 \%, 1 \%$ and $1.5 \% \mathrm{w} / \mathrm{v}$ were selected for optimization of formulation, homogenization speed of high, medium and low i. e 15,000 rpm, 10,000 rpm and 5,000 rpm was selected for trials. Solvent: antisolvent ratio is important for the stability of the drug crystals which in turn is important for the stability of the formulation. Hence, solvent: antisolvent 1:5, 1:10 and $1: 15$ ratios were selected for trials.

\section{Characterization of nanosuspensions (NSs)}

\section{Particle size and zeta potential}

Photon correlation spectroscopy (PCS) is a technique used to determine the mean particle size diameter (mean PCS diameter) and the width of the particle size distribution expressed as PDI [9]. The PCS and zeta potential measurements were performed using a malvern zetasizer nano ZS 90, malvern instruments (Malvern, UK). Before the measurements, samples were diluted with bidistilled water to a suitable scattering intensity and re-dispersed by handshaking, and then each sample was determined in triplicate.

The average particle size diameter and PDI were obtained after 20 runs. Zeta potential is the electric potential of a particle in a suspension. It is a parameter which is very useful for the assessment of the physical stability of colloidal dispersions. The surface charge generates a potential around the particle, which is at the highest near the surface and decays with distance into the medium. The samples were diluted with millipore water. Zeta potential of the formulation was determined by the average of the zeta potential from 25 runs in triplicate.

\section{Drug loading}

The drug loading of CUR was determined directly by measuring the concentration of drug in the NS. An aliquot of CUR NS was suitably diluted with methanol. The absorbance of the resulting solution was measured by UV-Vis spectrophotometer (Shimadzu 1800) at $420 \mathrm{~nm}$ against methanol as blank.

$$
\% \text { Drug loading }=\frac{\text { Actual drug }}{\text { Theoreticaldrug }} \times 100
$$




\section{Saturation solubility}

NSs tend to increase the solubility of poorly water-soluble drugs by improving their dissolution. The study was performed as cited by yan gao et al. [10]. In this study same volume of CUR dispersed in water and CUR NS were added separately into water and phosphate buffer $\mathrm{pH}$ 6.8. These mixtures were kept for magnetic stirring (Remi labs, India) for $48 \mathrm{~h}$. The mixture was then centrifuged and aliquot from the supernatant was suitably diluted with methanol. The absorbance of the resulting solution was measured by UV-Vis spectrophotometer (Shimadzu 1800) at $420 \mathrm{~nm}$ against methanol as blank $[10,11]$.

\section{pH-dependent solubility}

In this study acetate buffer $\mathrm{pH} 4$, buffer $\mathrm{pH} 1.2$ and phosphate buffers $\mathrm{pH} 6.8$ and 7.4 alone and along with solubilizers were screened to determine the solubility of the drug. An excess amount of CUR was added to $10 \mathrm{ml}$ of medium and placed on a thermostatically controlled shaker (orbital shaker, Remi labs, India) maintained at $37{ }^{\circ} \mathrm{C}$ for $24 \mathrm{~h}$ and the amount of drug dissolved was determined by UV-Vis spectrophotometer (Shimadzu 1800) $[10,11]$.

\section{In vitro drug release studies}

In this study various ratios of phosphate buffer $\mathrm{pH}$ 6.8: ethanol was screened to finalize the dissolution medium. The excess drug was added to these medium and subjected to magnetic stirring for $24 \mathrm{~h}$. An aliquot of the mixtures was further centrifuged (Remi labs, India) and supernatant was suitably diluted with ethanol. The amount of drug dissolved was determined using UV-Vis spectrophotometer (Shimadzu 1800).

In vitro drug release studies were carried out using dialysis-bag (molecular cut-off 12000-14000 KDa) method in which aliquot of the drug suspension was filled into a dialysis bag and transferred to dissolution medium, selected from earlier studies. The assembly was covered to protect the drug from light degradation and prevent evaporation of dissolution medium. The volume of the dissolution medium was maintained constant. Suitable aliquots were withdrawn at intervals of $1,2,3,4,5$, and $24 \mathrm{~h}$ [11].

\section{Pharmacokinetic study}

Male SD rats weighing about 250-350 g were obtained from Bharat serums and vaccines Ltd, Thane, India. Animal experiments were performed in compliance with the guide for the care and use of laboratory animals and the declaration announced by the committee for the purpose of control and supervision of experiments on animals, the government of India. Protocol for animal studies was approved by Institutional Animal Ethics Committee, for pharmacokinetic experiments using animals other than non-human primates [Approval Ref no: VESCOP/07/2015 by the Institutional Animals Ethics Committee (IAEC)].

\section{Drug administration and blood plasma collection}

The SD rats were housed under standard conditions and provided with fresh water and diet. Before the experimentation animals fasted overnight. Unformulated CUR and developed formulation were administered orally. Placebo was administered to the control group. SD rats weighing in the range of 250-350 g were taken and divided into 2 groups. The first group received an aliquot of developed NS formulation. The second group received plain CUR $S$.

Blood samples were withdrawn from the retro-orbital plexus using capillary at $30 \mathrm{~min}, 1,2,4,6 \mathrm{~h}$ time interval following the administration of the formulation. The blood was collected in EDTA tubes and then centrifuged at 15,000 rpm for $15 \mathrm{~min}$ to separate the plasma. The plasma was stored at $-20^{\circ} \mathrm{C}$ until the time of analysis [10].

\section{Sample preparation}

Before analysis, the plasma samples were thawed at ambient temperature. A suitable volume of plasma sample was mixed with distilled water and known concentration of internal standard (Emodin). The mixture was vortexed to ensure complete mixing of contents. It was extracted by adding the mixture of extracting reagent (ethyl acetate and methanol) followed by centrifugation. The upper organic layer was collected and the sample was again subjected to extraction. Organic layers were pooled, dried with a gentle flow of nitrogen gas and reconstituted in $200 \mu \mathrm{l}$ of mobile phase and subjected to HPLC analysis [10].

\section{Drug concentration analysis in vivo}

The HPLC method was set up to determine the drug concentration in plasma. The chromatographic separation was carried out by using HIQsil C18 column (4.6 mm X $150 \mathrm{~mm}$, particle size $5 \mu \mathrm{m}$ ) along with guard column HIQsil C18 (4.6 mm I.D. X $10 \mathrm{~mm}$ ). The column oven temperature was maintained at $40^{\circ} \mathrm{C}$.

The mobile phase consisted of a mixture of acetonitrile and 50 mmol orthophosphoric acids in a ratio of $48: 52 \mathrm{v} / \mathrm{v}$ with a flow rate of $1.5 \mathrm{ml} / \mathrm{min}$. The total run time was $18 \mathrm{~min}$ and the sample injection volume was set at $20 \mu \mathrm{l}$ with detection wavelength at 425 nm. Data acquisition was processed with ChromNAV CFR 1.14.01 (build 3) software. The pharmacokinetic parameters like Cmax and AUC were calculated from the mean drug concentration-time profile curve.

\section{RESULTS AND DISCUSSION}

\section{Screening of solvents}

Various water-miscible solvents were screened and the solvent in which maximum amount of CUR dissolved was selected as the solvent phase for further studies. Fig. 2 shows the solubility of CUR in different organic solvents. Ethyl acetate showed maximum solubilization of CUR as compared to others. With restrictions placed on the use of chlorinated solvents such as dichloromethane, it is reasonable to use ethyl acetate, a polar solvent, to provide an acceptable quality of product and commercially viable yields. The order of solubility of CUR in various solvent screened was found to be: ethyl acetate>ethanol>acetone.

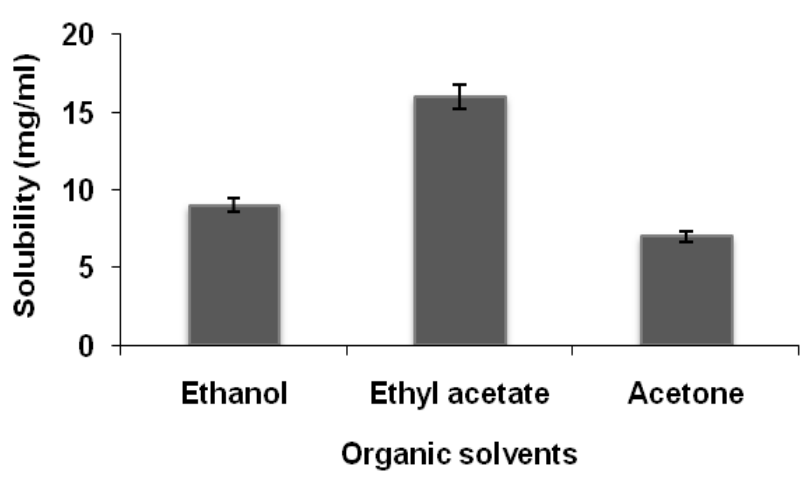

Fig. 2: Screening of organic solvents. (Data are given in mean $\pm S D)(n=3)$, SD: Standard deviation

\section{Screening of stabilizers}

The process of nanosizing is sensitive to the choice of the stabilizers as the stabilizers (polymeric and/or surfactant excipients) can prevent particle agglomeration and compensate the extra free energy of the freshly exposed surfaces. In this study, it was found that the drug was evenly dispersible in all the surfactants.

Table. 1 depicts the results of stabilizers screening studies. It was found that SLS formed a hard cake. A substantial increase in size was obtained with tween 80 . In case of TPGS, the particle size decreased with time but the size distribution became broad. P188 was the surfactant of choice because it showed less variability, smooth dispersion and stable formulation though the size distribution was broad. Fig. 3 shows the effect of concentration of P188 on the particle size of CUR. 
Table 1: Screening of stabilizers

\begin{tabular}{llll}
\hline Surfactants & $\mathbf{0}^{\text {th }}$ day & \multicolumn{1}{l}{$\mathbf{7}^{\text {th }}$ day } \\
\cline { 2 - 4 } & Particle size $(\mathbf{n m}) \mathbf{( m e a n} \pm$ SD) & $\begin{array}{l}\text { PDI } \\
\text { (mean } \pm \text { SD) }\end{array}$ & Particle size (nm) (mean \pm SD) \\
\hline SLS & $149.00 \pm 4.47$ & $0.278 \pm 0.009$ & Hard cake was observed. \\
Tween 80 & $34.85 \pm 0.79$ & $0.351 \pm 0.014$ & $256.00 \pm 9.96$ \\
TPGS & $780.10 \pm 18.78$ & $0.122 \pm 0.004$ & $519.00 \pm 13.63$ \\
P188 & $486.30 \pm 9.79$ & $0.650 \pm 0.009$ & $505.50 \pm 9.20$ \\
\hline
\end{tabular}

Data given in this table is presented as mean $\pm S D, n=3$, SD: Standard deviation.

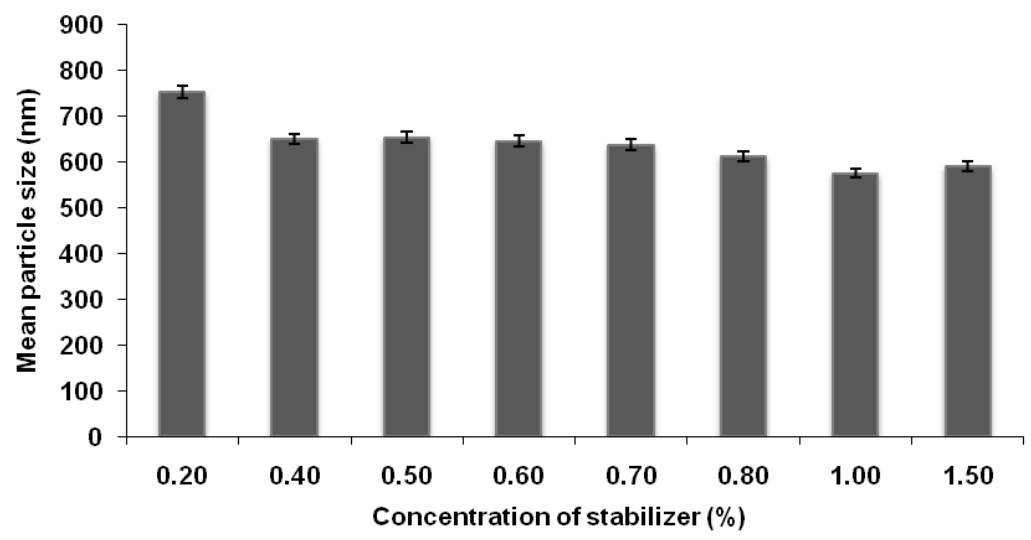

Fig. 3: Effect of the amount of stabilizer on the particle size distribution. (Data given in mean \pm SD) (n=3), SD: Standard deviation

Poloxamers are nonionic synthetic copolymers of polyethene oxide (PEO) and polypropylene oxide (PPO). PPO usually forms central hydrophobic core wherein methyl groups interact via van der walls forces with the substance undergoing solubilization. However, water solubility is due to PEO block, by hydrogen bonding interactions of ether oxygen with water molecules. Due to these interactions, poloxamers are readily soluble in polar and non-polar organic solvents. NSs prepared using $1 \% \mathrm{w} / \mathrm{v}$ P188 showed the average diameter of $575.8 \pm 10.36 \mathrm{~nm}$. With the increase in the concentration of the stabilizer up to $0.8 \% \mathrm{w} / \mathrm{v}$, particle size decreased followed by an increase in particle size as shown in fig. 2. NS prepared with P188 showed higher particle size and PDI with a smooth appearance which did not change significantly even after 7 d. Hence, P188 was selected for further studies wherein NSs were prepared varying its concentration.

\section{Optimization studies}

Results of optimization studies indicated that use of P188 at $1 \% \mathrm{w} / \mathrm{v}$ concentration conferred required stability to the formulation. Solvent: the anti-solvent ratio of 1:15 resulted in smaller particle size. The drug precipitating in a small amount of antisolvent than large amount happens to agglomerate with another precipitating molecule just in vicinity making a large particle which is not desirable. At 10,000 rpm and 15,000 rpm speed of $\mathrm{HSH}$, smaller particle size was obtained. $10,000 \mathrm{rpm}$ was selected as optimum speed because the increase in speed to $15,000 \mathrm{rpm}$ did not change the particle size significantly. In addition, it leads to discolouration of NS. This may be attributed to the temperature sensitivity of the drug. Thus surfactant concentration of $1 \% \mathrm{w} / \mathrm{v}$ with solvent: the antisolvent ratio of 1:15 and homogenization speed of 10,000 rpm followed by probe sonication was selected.

\section{Characterization of nanosuspensions}

\section{Particle size and zeta potential}

The particle size of NS has significance in the performance of oral drug delivery systems. It is presented as z-average diameter, basically, mean hydrodynamic diameter of the particles. Particle size measurement was required to confirm the production of the particles in nano-range. It has been observed that the particle size of the formulation was $596.5 \pm 5 \mathrm{~nm}$ with PDI of $0.233 \pm 0.010$ indicating that the formulation is not much polydispersed.
The magnitude of zeta potential is an indication of the repulsive force that is present in nanoparticles and is a key factor in predicting the long-term stability of colloidal dispersion system. Zeta potential in the range $\pm 30 \mathrm{mV}$ and $\pm 20 \mathrm{mV}$ considered ideal for electrostatic NS and steric stabilized NS respectively [7]. In the present study nonionic stabilizer P188 used which yields steric stabilized NS and hence the zeta potential value indicated that prepared NS is stable.

\section{Drug loading}

Drug loading for optimized formulation was found to be $98.25 \pm 0.81 \%$, as CUR was dissolved in solvent phase and precipitated by direct addition into antisolvent phase. Negligible loss of CUR was due to adhesion to processing containers.

\section{Saturation solubility}

The saturation solubility studies were carried out for both unprocessed pure drug and the optimized batch of NS. The plain drug showed solubility of $0.652 \pm 0.003 \mu \mathrm{g} / \mathrm{ml}$ in distilled water and $0.6916 \pm 0.002 \mu \mathrm{g} / \mathrm{ml}$ in $\mathrm{pH} 6.8$ buffer. NS showed a solubility of $2.217 \pm 0.027 \mu \mathrm{g} / \mathrm{ml}$ in distilled water and $3.186 \pm 0.016 \mu \mathrm{g} / \mathrm{ml}$ in $\mathrm{pH}$ 6.8 buffer. As shown in fig. 4 NS showed better solubility due to nanotisation and better wetting ability conferred by the surfactant. Due to HSH, new surfaces are formed, which leads to increase in an interfacial area of contact between drug and dissolution medium, leading to improved solubility.

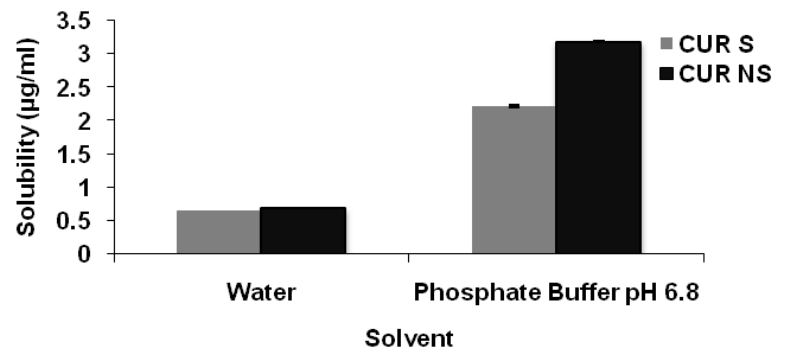

Fig. 4: Comparative saturation solubility studies, (Data given in mean \pm SD) $(n=3)$, SD: Standard deviation 


\section{pH-dependent solubility}

As shown in fig. 5 solubility of the drug increases with increase in $\mathrm{pH}$. It was observed that the drug possesses maximum solubility at $\mathrm{pH} 7.4$ followed by $\mathrm{pH}$ 6.8. At $\mathrm{pH} 7.4$ color of NS was changed from yellowish orange to dark orange. With due consideration of appearance and stability $\mathrm{pH} 6.8$ was considered for further studies.
As the solubility of the drug was very less, solublizers were needed. Solubilizers like SLS $0.5 \% \mathrm{w} / \mathrm{v}$ and $1 \% \mathrm{w} / \mathrm{v}$ did not help much insolubilization of the drug. Addition of ethanol to buffer in the ratio (50:50) showed better results.

A mixture containing ethanol and buffer having $\mathrm{pH} 6.8$ in the ratio 50:50 was selected as dissolution medium for further studies.

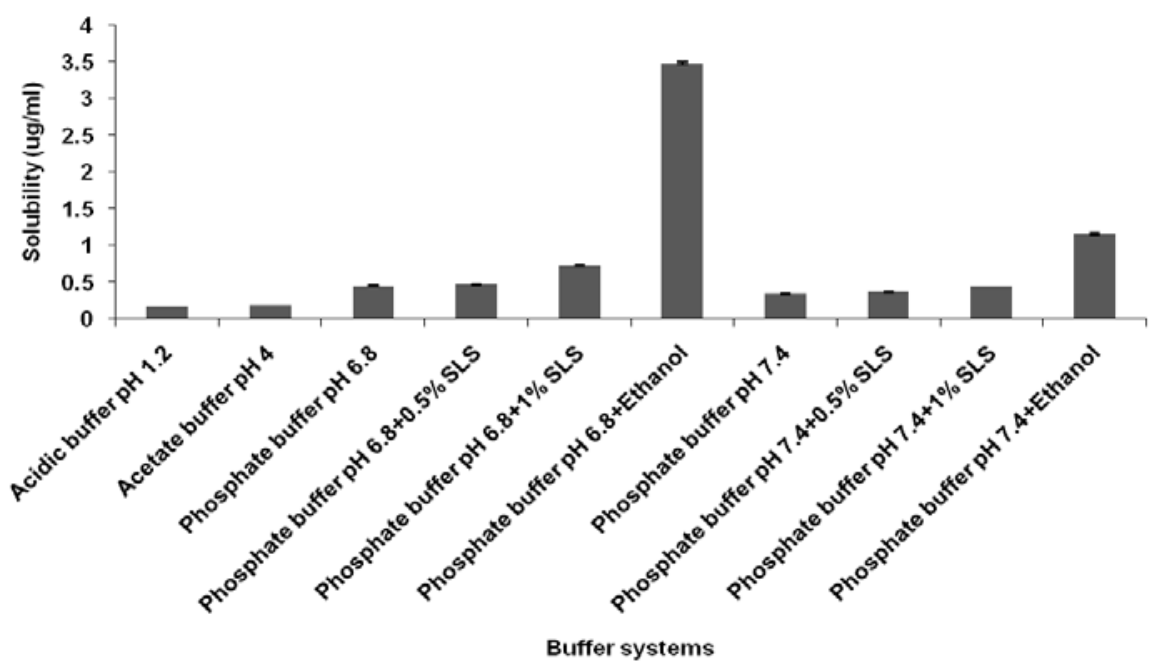

Fig. 5: pH-dependent solubility of CUR in various buffers, (Data given in mean \pm SD) $(n=3)$, SD: Standard deviation

\section{In vitro drug release}

Dissolution studies were performed over the period of $5 \mathrm{~h}$ using different dissolution media compositions. It was found that phosphate buffer pH 6.8 and ethanol, (50:50) gave maximum dissolution as shown in the table 2. The CUR release from the NS was compared with that of the CUR S. It was found that only $23.9 \%$ of the CUR was released from CUR S in water in comparison with $83.9 \%$ from the NS in $5 \mathrm{~h}$ as indicated in the fig. 6 . Similar results were obtained by Jyothi et al. [12].

Table 2: Screening of dissolution medium

\begin{tabular}{ll}
\hline Dissolution media & Concentration $(\boldsymbol{\mu g} / \mathbf{m l})(\mathbf{m e a n} \pm$ SD) \\
\hline Phosphate buffer pH 6.8+ethanol (90:10) & $0.984 \pm 0.037$ \\
Phosphate buffer pH 6.8+ethanol (80:20) & $1.020 \pm 0.031$ \\
Phosphate buffer pH 6.8+ethanol (70:30) & $1.310 \pm 0.048$ \\
Phosphate buffer pH 6.8+ethanol (60:40) & $2.410 \pm 0.0045$ \\
Phosphate buffer pH 6.8+ethanol (50:50) & $3.480 \pm 0.069$ \\
\hline
\end{tabular}

Data given in this table is presented as mean $\pm S D, n=3$, SD: Standard deviation.

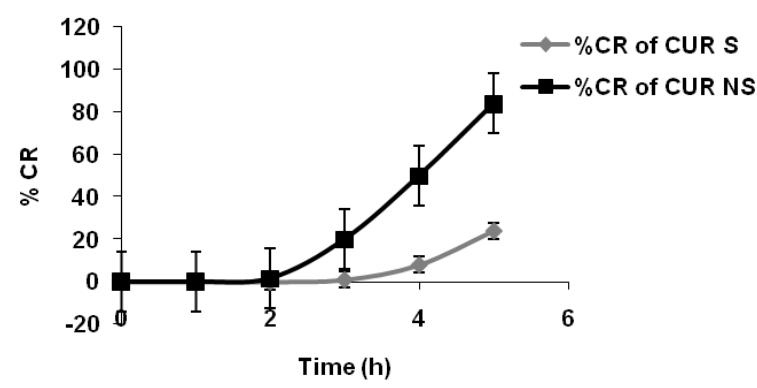

Fig. 6: \% cumulative drug release from CUR $S$ and CUR NS. (Data are given in mean $\pm S D)(n=3)$, SD: Standard deviation

\section{Pharmacokinetics studies}

The NS formulation was subjected to pharmacokinetic studies to evaluate various kinetic parameters such as drug concentration in plasma, AUC, $t_{\max }$ by HPLC method. CUR from NS attained maximum plasma concentration after half an hour from administration whereas plain CUR attained maximum concentration after $1 \mathrm{~h}$ of administration. Results of pharmacokinetic studies performed on SD rats after administration of CUR NS and CUR S are shown in fig. 7 and table 3 . The higher $C_{\max }$ and lower $t_{\max }$ value of CUR NS suggested that more CUR was absorbed into the blood after rapid dissolution into the gastrointestinal tract (GIT) in lesser time as compared to C-S. The $\mathrm{C}_{\max }$ and $\mathrm{AUC}_{0-6}$ value of CUR NS were approximately 8 times and 30 times higher than CUR S respectively. The relative bioavailability of CUR NS was high which means that the CUR gets absorbed rapidly from GIT due to elevated dissolution rate obtained by decreasing particle size [13]. Detailed investigation on the effect of reduced particle size on the dissolution of CUR studied by Sachin et al. [14]. Hence, nanotisation could significantly improve the bioavailability of CUR [15]. 


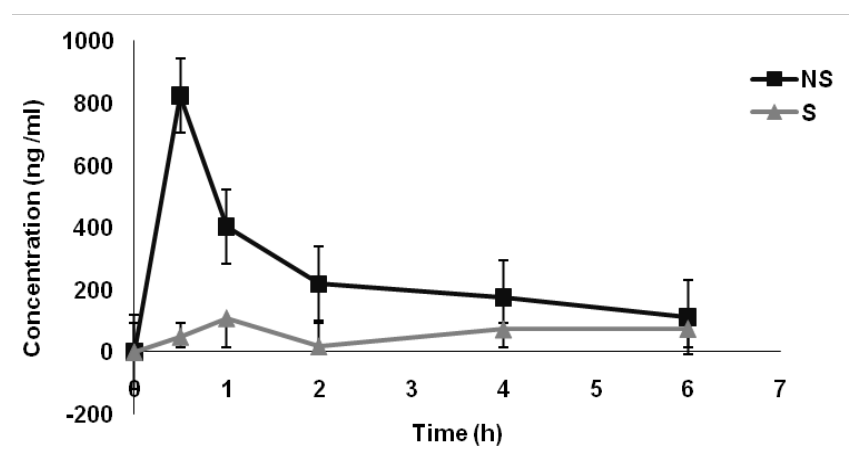

Fig. 6: Pharmacokinetic studies CUR $S$ and CUR NS. (Data are given in mean \pm SD) $(n=6)$, SD: Standard deviation

Table 3: Pharmacokinetic parameters

\begin{tabular}{llll}
\hline Formulation & $\mathbf{C}_{\max }(\mathbf{n g} / \mathbf{m l})(\mathbf{m e a n} \pm \mathbf{S D})$ & $\mathbf{A U C}_{\mathbf{0}-\mathbf{6}}(\boldsymbol{\mu} \mathbf{g} / \mathbf{m l} \mathbf{m} \mathbf{m i n})(\mathbf{m e a n} \pm \mathbf{S D})$ & $\mathbf{t}_{\max }(\mathbf{m i n})$ \\
\hline CUR NS & $823.39 \pm 167.21$ & $1503.45 \pm 136.58$ & 30 \\
CUR S & $108.55 \pm 20.48$ & $355.65 \pm 54.88$ & 60 \\
\hline
\end{tabular}

Data given in this table is presented as mean $\pm S D, n=6$, SD: Standard deviation.

\section{CONCLUSION}

The aim of the present work was the preparation and evaluation of CUR NS. CUR NS was prepared by precipitation with aid of HSH followed by probe sonication. Different surfactants, solvents, and solvent: antisolvent ratios were screened during formulation development. Ethyl acetate was selected as a solvent phase as it showed maximum solubility. Precipitation with aid of HSH followed by probe sonication resulted in formulation with less variability and more reproducibility. The optimized formula was arrived at, which contained 1\% w/v P188, 1:15 ratio of solvent: antisolvent with HSH at $10,000 \mathrm{rpm}$ followed by $10 \mathrm{~min}$ of probe sonication. The particle size distribution and PDI of CUR NS were $596.5 \pm 5 \mathrm{~nm}$ and $0.233 \pm 0.010$. Drug loading of the formulation was found to be $98.25 \pm 0.81 \%$. In vivo studies in SD rats indicated that NS readily gets absorbed compared to plain CUR. In short, NS delivery system was effective in enhancing the oral bioavailability of the drug. Bioavailability of drug can further be enhanced by modifying the metabolism of the absorbed drug.

\section{ACKNOWLEDGEMENT}

The authors would like to thanks All India Council for Technical Education (AICTE), New Delhi for supporting this work (Ref no: 8159/RIFD/RPS/Policy-4/2013-14). Authors are thankful to VAV Life science and BASF, Mumbai for providing gift samples. Our special thanks to Advanced Centre for Treatment, Research and Education in Cancer (ACTREC), Tata Memorial Centre, Navi-Mumbai for carrying out pharmacokinetic studies.

\section{AUTHORS CONTRIBUTIONS}

Experimental design, execution, data generation and writing of manuscript were done by Sneha Dekate. Support to draft manuscript design, data interpretation and corrections were done by Srinivas Bhairy. The design, guidance for work and manuscript review was done by Dr. (Mrs.) Rajashree Hirlekar.

\section{CONFLICT OF INTERESTS}

\section{Declared none}

\section{REFERENCES}

1. Manish M, Sundaramoorthy S. Anticancer herbal drugs and their improvement through novel drug delivery approaches. Appl Biol Res 2012;15:1-20.
2. Nidhi A, Chandana M, Chakraborthy GS. Natural herbs as anticancer drugs. Int J PharmTech Res 2012;4:1142-53.

3. Rajashree H, Vilasrao K. Preparation and characterization of inclusion complexes of carvedilol with methyl- $\beta$-cyclodextrin. J Incl Phenom Macrocycl Chem 2009;63:219-24.

4. Rajashree H, Harshal G, Vilasrao K. Solid-state characterization of the inclusion complex of valsartan with methyl $\beta$-cyclodextrin. J Incl Phenom Macrocycl Chem 2009; 65:377-83.

5. Rajashree H, Harshal G, Vilasrao K. Solid lipid nanoparticles and nanostructured lipid carriers: a review. Curr Drug Ther 2011;6:240-50.

6. Samar AA, Maha AH, Ali SA, Kadria AE. Nanosuspension: an emerging trend for bioavailability enhancement of etodolac. Int J Polym Sci 2015;1-16. http://dx.doi.org/10.1155/2015/ 938594.

7. Vandana BP, Abhijit AD, Kulkarni RM. Nanosuspensions: a promising drug delivery strategy. J Pharm Pharmacol 2004;56:827-40.

8. Preetha A, Ajaikumar BK, Robert AN, Bharat BA. Bioavailability of curcumin: problems and promises. Mol Pharm 2007;4:807-18.

9. Muller RH. Colloidal carriers for controlled drug delivery and targeting. Modification, characterization, and in vivo distribution. J Controlled Release 1992;18:279-80.

10. Yan G. Preparation, characterization, pharmacokinetics, and tissue distribution of curcumin nanosuspension with TPGS as a stabilizer. Drug Dev Ind Pharm 2010;36:1225-34.

11. Rupali LS, Nilesh K. Formulation and evaluation of a nanosuspension delivery system for simvastatin. Int J Pharm Sci Nanotech 2014;7:2459-76.

12. Jeevana JB, Sravani R. Development of curcumin nanocrystals and evaluation of GIT absorption efficiency in copmparison with curcumin and turmeric powder. World J Pharm Pharm Sci 2016;5:1990-2003.

13. Hintz RJ, Johnson KC. The effect of particle size distribution on dissolution rate and oral absorption. Int J Pharm 1989;51:9-17.

14. Sachin KS, Jasmine K, Palak B, Sarvi YR, Parth S, Deepak G, et al. Formulation of curcumin nanosuspension using boxbehnken design and study of the impact of drying techniques on its powder characteristics. Asian J Pharm Clin Res 2017; 10:43-51.

15. Smita SA, Malsane ST, Saudagar RB. Nanosuspension: an overview. Int J Curr Pharm Res 2017;9:19-23. 